Bundesgesundheitsbl 2021 · 64:802-811 https://doi.org/10.1007/s00103-021-03358-1 Eingegangen: 22. Januar 2021

Angenommen: 20. Mai 2021

Online publiziert: 22. Juni 2021

c c Der/die Autor(en) 2021

\section{Ina Nitschke ${ }^{1,2} \cdot$ Sebastian Hahnel ${ }^{1}$}

' Poliklinik für Zahnärztliche Prothetik und Werkstoffkunde, Universitätsklinikum Leipzig, Universität Leipzig, Leipzig, Deutschland

${ }^{2}$ Klinik für Allgemein-, Behinderten- und Seniorenzahnmedizin, Universität Zürich, Zürich, Schweiz

\title{
Zahnmedizinische Versorgung älterer Menschen: Chancen und Herausforderungen
}

\section{Einleitung}

Der Anteil älterer Menschen an der Gesamtbevölkerung ist steigend [1], sodass sich die sozialen Versorgungssysteme immer größeren Herausforderungen stellen müssen. In Deutschland haben alle Bürgerinnen und Bürger das Recht auf eine bedarfsgerechte gesundheitliche Versorgung. Dieses Recht ist Bestandteil der Menschenrechte (UN-Behindertenrechtskonvention, 26.03.2009 für Deutschland ratifiziert [2]), welche sicherstellen, dass das individuell erreichbare Höchstmaß an Gesundheit ohne Diskriminierung aufgrund einer Behinderung zur Verfügung steht. Hier sind auch Ältere sowie Kinder, Gebrechliche und Pflegebedürftige eingeschlossen. Eine bedarfsgerechte gesundheitliche Versorgung beinhaltet die gesundheitliche Prävention, Behandlung und Betreuung. Die ambulanten und stationären Versorgungssysteme sind bei nachlassender funktioneller Kapazität innerhalb der heterogenen Bevölkerungsgruppe der Seniorinnen und Senioren gefordert Versorgungsangebote bereitzuhalten, die den Notwendigkeiten der sich stetig und manchmal sehr schnell ändernden Ansprüche angepasst sind.

In dieses Versorgungssystem, welches durch die politischen Organe der Gesetzgebung in der Makroebene definiert, durch die Krankenversicherer in der Mesoebene umgesetzt und durch die im Gesundheitssystem Beschäftigten in der Mikroebene ausgeführt wird, gehört auch die Behandlung oraler Erkrankungen. Die Mundgesundheit ist durch präventive Maßnahmen zu schützen oder wiederherzustellen, wenn Defizite auftreten.

Durch Präventionsmaßnahmen verfügen ältere Menschen über deutlich mehr Zähne als noch vor 20 Jahren; Zahnlosigkeit ist seltener geworden und Zahnersatz kann auf hohem Qualitätsniveau hergestellt werden. Dies ist jedoch nur möglich, wenn nach einer partizipativen Therapieentscheidung eine Behandlung bei entsprechend belastbaren und kooperativen Seniorinnen und Senioren erfolgt. Diese Voraussetzungen findet die Zahnärztin oder der Zahnarzt in der Regel bei den funktionell nicht eingeschränkten fitten älteren Menschen. Bei gebrechlichen oder pflegebedürftigen Seniorinnen und Senioren präsentieren sich jedoch andere Herausforderungen, da die Folgen körperlicher und kognitiver Einschränkungen die zur Verfügung stehenden Therapiemöglichkeiten stark limitieren. Im Gegensatz zu Seniorinnen und Senioren ohne Pflegebedarf (SoP) weisen Seniorinnen und Senioren mit Pflegebedarf (SmP) eine schlechtere Mundgesundheit auf [3-5]. Daher sind auch andere Personen - Angehörige, gesetzliche Betreuende, Pflegepersonal, Ärztinnen und Ärzte - zunehmend in die zahnmedizinische Versorgung einzubeziehen. Das Gesundheitswesen ist gefordert, auch bei schwierigen Lebensumständen eine bestmögliche Mundgesundheit zu ermöglichen. Eine gute Mundgesundheit, verbunden mit infektionsfreier Mundhöhle und passendem Zahnersatz, trägt zur Lebensqualität, Vermeidung von allgemeinmedizini- schen Krankheiten und Komplikationen bei Seniorinnen und Senioren bei.

In dem Beitrag wird die Heterogenität der älteren Menschen hinsichtlich ihrer Mundgesundheit beschrieben und die sich daraus ergebenden Problemfelder für eine zahnmedizinische Betreuung werden dargestellt. Die Differenzen innerhalb der Mundgesundheit werden an zahnmedizinischen Daten einer bevölkerungsrepräsentativen Studie für Menschen mit und ohne Pflegbedarf erläutert. Verschiedene strukturelle Probleme werden benannt und Lösungsvorschläge gemacht, um chancengleiche Anbindung an eine zahnmedizinische Versorgungsstruktur zu etablieren.

\section{Lebensphasen als Herausforderung}

Der Alterungsprozess gestaltet sich mit dem Auftreten von chronischen Erkrankungen sehr unterschiedlich. Dabei sind 3 Phasen $\mathrm{zu}$ differenzieren, wobei sich an die erste fitte und zweite gebrechliche Phase oftmals eine dritte pflegebedürftige Lebensphase anschließt. Relevant sind in diesem Kontext insbesondere die Übergänge - also von fit zu gebrechlich sowie von gebrechlich zu pflegebedürftig. Oftmals durchlaufen Seniorinnen und Senioren alle 3 Phasen, wobei sie unterschiedliche Unterstützungssysteme benötigen. Hierbei ist nicht allein die Versorgung innerhalb der jeweiligen Phase relevant. Vielmehr besteht die Herausforderung darin, den Übergang in die nächste Phase möglichst hinauszuzögern und gut vorzubereiten. Dabei ist es die 
Tab. 1 Erkrankungen des Mundes, Zahnersatz und Inanspruchnahmeverhalten zahnärztlicher Leistungen bei älteren Seniorinnen und Senioren (75-100 Jahre) aufgefächert nach Pflegebedarf (Fünfte Deutsche Mundgesundheitsstudie, Erhebung 2014; [5])

\begin{tabular}{|c|c|c|c|}
\hline & Ohne Pflegestufe & Mit Pflegestufe & Gesamt \\
\hline Mundschleimhautveränderungen & $n=877$ & $n=256$ & $n=1133$ \\
\hline Karzinom (\%) & 0,2 & 0,0 & 0,2 \\
\hline Leukoplakie (\%) & 0,8 & 0,5 & 0,8 \\
\hline Erythroplakie (\%) & 0,6 & 1,0 & 0,7 \\
\hline Oraler Lichen planus (\%) & 0,1 & 0,7 & 0,2 \\
\hline Candida (\%) & 0,1 & 0,0 & 0,1 \\
\hline Raucherkeratose (\%) & 0,2 & 0,0 & 0,2 \\
\hline Prothesenbedingte Veränderung (\%) & 3,7 & 6,9 & 4,4 \\
\hline Sonstiges (\%) & 9,8 & 3,6 & 8,4 \\
\hline $\begin{array}{l}\text { Parodontale Erkrankungen } \\
\text { (basierend auf Full Mouth Protocol) }\end{array}$ & $n=642$ & $n=119$ & $n=761$ \\
\hline BOP (\%) & 43,2 & 64,3 & 46,5 \\
\hline Mittlere ST (mm) & 2,8 & 2,8 & 2,8 \\
\hline Mittlerer AV (mm) & 3,9 & 4,4 & 4,0 \\
\hline$C P I$ & $n=611$ & $n=111$ & $n=722$ \\
\hline Grad 0, 1, 2 (\%) & 17,5 & 30,0 & 19,4 \\
\hline Grad 3 (ST 4-5 mm; \%) & 53,3 & 35,1 & 50,5 \\
\hline Grad 4 (ST $\geq 6$ mm; \%) & 29,2 & 34,9 & 30,1 \\
\hline CDC/AAP Fallklassifikation & $n=544$ & $n=96$ & $n=640$ \\
\hline Keine/milde Parodontitis (\%) & 8,5 & 18,3 & 10,0 \\
\hline Moderate Parodontitis (\%) & 47,7 & 34,1 & 45,7 \\
\hline Schwere Parodontitis (\%) & 43,7 & 47,6 & 44,3 \\
\hline Anzahl fehlender Zähne & $n=877$ & $n=256$ & $n=1133$ \\
\hline Ohne Weisheitszähne & 16,5 & 22,4 & 17,8 \\
\hline Mit Weisheitszähnen & 20,2 & 26,3 & 21,6 \\
\hline Zahnlosigkeit & $n=877$ & $n=256$ & $n=1133$ \\
\hline Ober- und Unterkiefer (\%) & 26,7 & 53,7 & 32,8 \\
\hline Oberkiefer (\%) & 42,3 & 63,6 & 47,1 \\
\hline Unterkiefer (\%) & 28,1 & 55,8 & 34,4 \\
\hline Art des Zahnersatzes & $n=877$ & $n=256$ & $n=1133$ \\
\hline Kein Zahnersatz (\%) & 8,1 & 5,0 & 7,4 \\
\hline Abnehmbar (\%) & 51,7 & 77,1 & 57,4 \\
\hline Festsitzend (\%) & 23,1 & 8,3 & 19,8 \\
\hline Abnehmbar und festsitzend (\%) & 17,1 & 9,6 & 15,4 \\
\hline Inanspruchnahmeverhalten & $n=845$ & $n=254$ & $n=1099$ \\
\hline Beschwerdeorientiert (\%) & 31,8 & 61,3 & 38,4 \\
\hline Kontrollorientiert (\%) & 68,2 & 38,7 & 61,6 \\
\hline \multicolumn{4}{|c|}{$\begin{array}{l}\text { AV Attachmentverlust (Verlust an Zahnhalteapparat; Sondierungstiefe plus Ausmaß des Zahnfleisch- } \\
\text { rückganges), BOP „,bleeding on probing“ (Angaben zum Zahnfleischbluten beim Sondieren), CDC/AAP- } \\
\text { Fallklassifikation Fallklassifikation der Centers for Disease Control and Prevention/American Academy } \\
\text { of Periodontology, CPI Community Periodontal Index }\end{array}$} \\
\hline
\end{tabular}

tägliche Herausforderung der Zahnmedizin und anderer im Gesundheitswesen tätiger Akteure, die Mundgesundheit innerhalb dieser Lebensphasen aufrechtzuerhalten.

\section{Mundgesundheit älterer Menschen mit und ohne Pflegebedarf}

Die im Jahr 2016 veröffentlichte Fünfte Deutsche Mundgesundheitsstudie (DMS V; [6]) hat 20142 Gruppen der Seniorinnen und Senioren einbezogen (jüngere der Altersgruppe 65-74 Jahre, $n=1042$, und ältere der Altersgruppe 75-100 Jahre, $n=1133$ ). In dieser für Deutschland repräsentativen epidemiologischen Erhebung wurden in der Gruppe der älteren Seniorinnen und Senioren (äS), der Bevölkerung entsprechend, auch ältere Senioren mit Pflegebedarf (äSmP $N=256$ ) in die Studie aufgenommen [5]. Bei der Interpretation der Daten ist zu berücksichtigen, dass sowohl Menschen mit ausgeprägter Demenz als auch Menschen in einer Palliativsituation nicht in die Studie eingeschlossen wurden. Die Daten der äSmP innerhalb DMS V [5] dürften somit die Realität etwas beschönigen. Auch sind Aussagen mit Vergleichen zwischen den DMS III, IV und V durch die zeitlich aufeinander aufbauenden Querschnittsstudien limitiert.

\section{Erkrankungen der Mundschleim- haut}

Prothesenbedingte Mundschleimhautveränderungen (z.B. Druckstellen, Ulzerationen, Reizfibrome) treten bei den äSmP (6,9\%) im Vergleich zu älteren Senioren ohne Pflegebedarf (äSoP) fast doppelt so häufig auf. Bei äSmP wurde 7-mal häufiger als bei äSoP ein oraler Lichen planus diagnostiziert, wobei hier die insgesamt geringen Fallzahlen berücksichtigt werden sollten ([5]; - Tab. 1).

\section{Parodontale Erkrankungen}

Keine bzw. eine milde Parodontitis trat bei $18,3 \%$ der äSmP auf und damit mehr als doppelt so häufig wie bei äSoP $(8,5 \%)$. Der gingivale Entzündungszustand („bleeding on probing“) war bei den äSmP deutlich höher als bei äSoP. Trotz verringerter Zahnzahl bei den äSmP trat an 64,3\% der untersuchten Stellen eine Blutung auf; hingegen wurde eine Entzündung der Gingiva bei den äSoP nur an 43,2\% der untersuchten Stellen registriert ([5]; - Tab. 1).

\section{Zahnverlust und Zahnersatz}

Die mittlere Anzahl der fehlenden Zähne betrug bei den 65 - bis 74 -Jährigen 
in den Jahren 1997, 2005 und 2014 jeweils 17,6 Zähne [7], 14,2 Zähne [8] und 11,1 Zähne ([9]; bezogen auf 28 Zähne). ÄSoP haben durchschnittlich 11,8 Zähne, äSmP hingegen nur noch 5,7 Zähne. ÄSmP fehlen unter Berücksichtigung der Weisheitszähne durchschnittlich 26,3 Zähne (ohne Weisheitszähne: 22,4 Zähne) und damit 6,1 Zähne (ohne Weisheitszähne: 5,9 Zähne) mehr als bei äSoP ([5]; • Tab. 1).

Durch den Rückgang des Zahnverlustes hat sich der Anteil zahnloser jüngerer Seniorinnen und Senioren seit 1997 [7] von $24,8 \%$, etwas über $22,6 \%$ im Jahr 2005 [8] bis hin zu 12,4\% im Jahr 2014 [9] halbiert. $32,8 \%$ aller äS sind zahnlos ([10]; äSmP: 53,7\%, äSoP: 26,7\%; [5]). Mehrals 3 Viertel der äSmP sind abnehmbar prothetisch versorgt (äSoP: 51,7\%; - Tab. 1).

Zusammenfassend ist festzustellen, dass äSmP eine schlechtere Mundgesundheit als äSoP aufweisen [5]. Daher ist es notwendig, die zahnmedizinische Betreuung für die ambulant und stationär Pflegedürftigen zu verbessern.

\section{Oralgeriatrisches Assessment bei Gebrechlichkeit oder Pflegebedürftigkeit}

Im Jahr 2019 meldeten die Pflegekassen in Deutschland 4.251.638 Menschen mit Pflegebedürftigkeit (davon 3.141.471 ambulant pflegebedürftig; [11]). Das Statistische Bundesamt verweist auf 4.127.605 Pflegebedürftige, davon sind $62,3 \%$ Frauen und 3.309.288 ambulant pflegebedürftig [12]. Es ist im Gegensatz zu den allgemeinmedizinisch Gesunden eine Herausforderung, ältere Menschen mit Multimorbidität und Polymedikation zahnärztlich zu behandeln. Vergleichbar mit Assessments in der Geriatrie sollte es auch in der Seniorenzahnmedizin Standard werden, den älteren Menschen unter zahnmedizinischen Aspekten einzuschätzen, die zahnmedizinische Belastbarkeit festzulegen und diese zu Beginn einer neuen Behandlungsmaßnahme zu reevaluieren. Als oralgeriatrisches Assessmentelement steht dabei die zahnmedizinische funktionelle Kapazität (ZFK) mit den Parametern Therapiefähigkeit, Mundhygienefähigkeit und

Bundesgesundheitsbl $2021 \cdot 64: 802-811$ https://doi.org/10.1007/s00103-021-03358-1 (c) Der/die Autor(en) 2021

\section{Nitschke $\cdot$ S. Hahnel}

\section{Zahnmedizinische Versorgung älterer Menschen: Chancen und Herausforderungen}

\section{Zusammenfassung}

Die Mundgesundheit der Bevölkerung in Deutschland konnte in den letzten Jahren verbessert werden; allerdings profitierten ältere und insbesondere gebrechliche sowie pflegebedürftige Menschen nicht adäquat von dieser Entwicklung. Dabei kann eine gute Mundgesundheit relevant dazu beitragen, die Herausforderungen bei Gebrechlichkeit und Pflegebedürftigkeit besser zu bewältigen. Der eingeschränkte Zugang zur zahnmedizinischen Betreuung, die manchmal eingeschränkte Kooperativität sowie die schlechtere Mundpflege in dieser Bevölkerungsgruppe erhöhen das Risiko für Karies, Parodontalerkrankungen, Zahnverlust und Zahnlosigkeit im Vergleich zur Durchschnittsbevölkerung.

Der vorliegende Beitrag gibt eine Übersicht über die zahnmedizinische Situation älterer Menschen anhand bereits publizierter Daten aus der bevölkerungsrepräsentativen Fünften Deutschen Mundgesundheitsstudie (DMS V), die im Jahr 2014 erhoben wurden. Die mittlere Anzahl der fehlenden Zähne betrug bei den 65- bis 74-Jährigen 11,1 Zähne. Bei älteren Seniorinnen und Senioren
(75-100 Jahre) mit Pflegebedarf (äSmP) war die Mundgesundheit schlechter als bei denjenigen ohne Pflegebedarf (äSoP). So hatten äSoP durchschnittlich 11,8 Zähne, äSmP dagegen nur 5,7 Zähne. Der Anteil zahnloser 65- bis 74-Jähriger hatte sich seit 1997 halbiert auf $12,4 \%$. Bei den äS waren $32,8 \%$ zahnlos (äSmP: 53,7\%, äSoP: $26,7 \%)$. Mehr als $75 \%$ der äSmP waren abnehmbar prothetisch versorgt (äSoP: 51,7\%). Vor diesem Hintergrund werden in diesem Beitrag Schnittstellen benannt, an denen eine chancengleiche Anbindung dieser Bevölkerungsgruppen an eine zahnmedizinische Versorgungsstruktur etabliert werden könnte. Diese beinhalten akutgeriatrische Krankenhausstationen und die Entwicklung weitergehender Konzepte in der aufsuchenden Versorgung zur besseren Versorgung der Betroffenen und zur Erleichterung der Pflege.

\section{Schlüsselwörter}

Mundgesundheit - Geriatrische Zahnmedizin . Menschen mit Pflegebedarf · Chancengleicher Zugang · Inanspruchnahmeverhalten

\section{Dental care for older people: opportunities and challenges}

\section{Abstract}

The oral health of the population in Germany has improved in recent years; however, older people, especially those with frailty and in need of care, have not benefited adequately from this development. Yet, good oral health can make a relevant contribution to better coping with the challenges of frailty and the need for long-term care. Limited access to dental care, and in some cases reduced cooperation and poorer oral hygiene, increases the risk of caries, periodontal diseases, and loss of teeth as well as leads to a higher prevalence of edentulism in comparison to the general population. This paper provides an overview of the dental situation of older people based on previously published data from the population representative Fifth German Oral Health Study (DMS V), which was collected in 2014. The mean number of missing teeth was 11.1 among 65 - to 74 -year-olds. Older seniors (75-100 years) with care needs (äSmP) had worse oral health than those without care needs (äSoP). Thus, on average, äSoP had 11.8 teeth, whereas äSmP had only 5.7 teeth. The proportion of edentulous 65-74year-olds had halved since 1997 to $12.4 \%$. Among the äS, 32.8\% were edentulous (äSmP: $53.7 \%$, äSoP: $26.7 \%$ ). More than $75 \%$ of the äSmP had removable prosthetic restorations (äSoP: $51.7 \%$ ). Against this background, this paper identifies interfaces where an equal opportunity connection of these population groups to a dental care structure could be established. These include acute geriatric hospital wards and the development of more far-reaching concepts in outreach care to improve care for those affected and to facilitate care.

\section{Keywords}

Oral health · Geriatric dentistry $\cdot$ People with care requirements - Equal opportunities . Take-up of dental services 
Tab. 2 Kriterien zur individuellen Einschätzung der zahnmedizinischen funktionellen Kapazität (ZFK). Oralgeriatrisches Assessment [14, 15]

\begin{tabular}{|c|c|}
\hline $\begin{array}{l}\text { Parameter } \\
\text { der ZFK }\end{array}$ & Kriterien zur individuellen Einschätzung \\
\hline \multirow{13}{*}{$\begin{array}{l}\text { Therapie- } \\
\text { fähigkeit }\end{array}$} & Behandlungsort \\
\hline & Transportfähigkeit in zahnärztliche Praxis \\
\hline & Umsetzen in den Behandlungsstuhl möglich \\
\hline & Lagerungseinschränkung \\
\hline & Möglichkeit der Diagnostik \\
\hline & Längere Mundöffnungsphasen \\
\hline & Risiko für allgemeinmedizinische Zwischenfälle \\
\hline & Risiko für Medikamenteninteraktion \\
\hline & Risiken während der Behandlung \\
\hline & Verständnis von Anweisungen/Wiedergabe von Sachinhalten \\
\hline & Nachsorgekompetenz \\
\hline & Manuelle Geschicklichkeit \\
\hline & Adaptationsfähigkeit \\
\hline \multirow{11}{*}{$\begin{array}{l}\text { Mundhygiene- } \\
\text { fähigkeit }\end{array}$} & Greiffähigkeit \\
\hline & Handkraft \\
\hline & Manuelle Geschicklichkeit bei der Durchführung der Mundhygiene \\
\hline & Sehvermögen \\
\hline & Durchführung der häuslichen Mundhygiene \\
\hline & Schwierigkeitsgrad der Reinigbarkeit der Mundhöhle \\
\hline & Verstehen von Anweisungen und Ratschlägen \\
\hline & Erhaltene Ratschläge umsetzen \\
\hline & Nachsorgekompetenz \\
\hline & Fremdputzer vorhanden zur Überwachung und Durchführung der Mundhygiene \\
\hline & Fähigkeit und Möglichkeit, Mundhygieneprodukte einzukaufen bzw. zu erhalten \\
\hline \multirow{8}{*}{$\begin{array}{l}\text { Eigen- } \\
\text { verantwort- } \\
\text { lichkeit }\end{array}$} & Erkennen von Problemen \\
\hline & Willensäußerung \\
\hline & Entscheidungsfähigkeit \\
\hline & Besuchsverhalten \\
\hline & Organisationsfähigkeit \\
\hline & Nachsorgekompetenz \\
\hline & Verantwortungsträger (Vollmacht, Patientenverfügung) \\
\hline & Gerichtlich eingesetzte Betreuung (Deutschland)/Beistand (Schweiz) \\
\hline
\end{tabular}

Tab. 3 Bewertung der individuellen Belastbarkeit im Rahmen der zahnmedizinischen funktionellen Kapazität auf Grundlage der Beurteilung der Parameter Therapiefähigkeit, Mundhygienefähigkeit und Eigenverantwortlichkeit [13-16]. Der am schlechtesten bewertete Parameter bestimmt die vergebene Belastbarkeitsstufe (BS)

\begin{tabular}{llll}
$\begin{array}{l}\text { Belastbarkeits- } \\
\text { stufe (BS) }\end{array}$ & Therapiefähigkeit & $\begin{array}{l}\text { Mundhygiene- } \\
\text { fähigkeit }\end{array}$ & Eigenverantwortlichkeit \\
\hline 1 & Normal & Normal & Normal \\
2 & Leicht reduziert & Leicht reduziert & \\
3 & Stark reduziert & Stark reduziert & Reduziert \\
4 & Keine & Keine & Keine
\end{tabular}

Eigenverantwortlichkeit zur Verfügung [13-15]. Jeder einzelne Parameter wird nach dem Anamnesegespräch mithilfe der benannten Kriterien von zahnärztlicher Seite bewertet (• Tab. 2). Innerhalb der 3 Parameter werden die Patientin oder der Patient in einer 4-stufigen Einteilung hinsichtlich der Belastbarkeit betrachtet. Dabei ist Stufe 1 die beste (normale Belastbarkeit) und Stufe 4 die schlechteste (keine Belastbarkeit). Beim Parameter Eigenverantwortlichkeit erfolgt eine Einteilung nur in 3 Stufen: normal (Stufe 1), reduziert (Stufe 3) oder gar nicht eigenverantwortlich (Stufe 4; - Tab. 3). Für den Gesamtscore, also die Festlegung der Belastbarkeitsstufe (BS) der Patientin oder des Patienten (BS 1, BS 2, BS 3, BS 4), ist der am schlechtesten bewertete Parameter ausschlaggebend.

Diese BS ist Ausdruck der ZFK. Dieses oralgeriatrische Assessment hilft Zahnärztinnen und Zahnärzten im partizipativen Therapieentscheidungsprozess und der zahnmedizinischen Betreuung, da die Belastbarkeit die begrenzende Komponente ist. Dabei besteht die Herausforderung darin, abzuschätzen, wie stark und in welchem Zeitraum sich die Belastbarkeit der zu Behandelnden verändern wird. Zudem können spezielle Behandlungsverfahren, wie z.B. das Duplizieren von Prothesen oder eine Planung von Zahnersatz nach dem g3S-Prinzip (simpel, stabil und solide), abgeleitet werden [15].

Die DMS V konnte zeigen, dass 90,2 \% der äSoP und 47,8\% der äSmP normal oder leicht reduziert therapiefähig waren. Somit sind fast die Hälfte der äSmP unter zahnmedizinischen Aspekten gut $\mathrm{zu}$ therapieren, ein Sachverhalt, der die Heterogenität der Pflegebedürftigen deutlich unterstreicht. Bei den äSmP ist nur etwa jeder Fünfte $(22,5 \%)$ in der Lage, eine normale Mundhygiene durchzuführen (äSoP: 63,1\%). Außerdem waren $17,4 \%$ der äSmP nicht mehr in der Lage, eigenverantwortlich Therapieentscheidungen $\mathrm{zu}$ treffen oder eigenständig einen Zahnarzttermin $\mathrm{zu}$ organisieren bzw. wahrzunehmen (äSoP: $0 \%$; - Tab. 4). An dieser Stelle sei noch einmal darauf hingewiesen, dass Menschen mit intensivem Pflegebedarf aus der Studie ausgeschlossen waren. BS 3 
Tab. 4 Zahnmedizinische funktionelle Kapazität (ZFK) bei älteren Seniorinnen und Senioren (75-100 Jahre), aufgefächert nach Pflegebedarf. Diezahnmedizinische funktionelle Kapazität wird in den Belastbarkeitsstufen (BS) 1-4 ermittelt (Fünfte Deutsche Mundgesundheitsstudie, Erhebung 2014; [16])

\begin{tabular}{|c|c|c|c|}
\hline & $\begin{array}{l}\text { Ohne Pflegestufe } \\
877\end{array}$ & Mit Pflegestufe & Gesamt \\
\hline$n$ & 877 & 256 & \\
\hline \multicolumn{4}{|l|}{ Therapiefähigkeit (\%) } \\
\hline Normal & 64,6 & 26,1 & 55,9 \\
\hline Leicht reduziert & 25,6 & 21,7 & 24,7 \\
\hline Stark reduziert & 9,8 & 43,7 & 17,5 \\
\hline Keine & 0,0 & 8,7 & 1,9 \\
\hline \multicolumn{4}{|c|}{ Mundhygienefähigkeit (\%) } \\
\hline Normal & 63,1 & 22,5 & 53,9 \\
\hline Leicht reduziert & 31,7 & 34,9 & 32,4 \\
\hline Stark reduziert & 5,3 & 33,2 & 11,6 \\
\hline Keine & 0,0 & 9,4 & 2,1 \\
\hline \multicolumn{4}{|c|}{ Eigenverantwortlichkeit (\%) } \\
\hline Normal & 88,7 & 41,0 & 77,9 \\
\hline Reduziert & 11,3 & 41,5 & 18,2 \\
\hline Keine & 0,0 & 17,4 & 3,9 \\
\hline \multicolumn{4}{|c|}{ Belastbarkeitsstufe (BS; \%) } \\
\hline BS 1 - voll belastbar & 56,4 & 17,5 & 47,6 \\
\hline BS 2 - leicht reduziert & 27,6 & 15,7 & 24,9 \\
\hline BS 3 - stark reduziert & 16,0 & 48,9 & 23,4 \\
\hline BS 4 - nicht belastbar & 0,0 & 17,9 & 4,0 \\
\hline
\end{tabular}

und 4 werden dementsprechend tatsächlich einen höheren Anteil bei den äSmP im Alltag ausmachen [16].

\section{Zahnärztliche Inanspruchnahme}

\section{Inanspruchnahmeverhalten zahnmedizinischer Leistungen}

Ärztliche Leistungen werden von fast allen Seniorinnen und Senioren in Anspruch genommen. Dahingegen sinkt die Inanspruchnahme der zahnärztlichen Leistungen: bei den Frauen von zunächst noch $80 \%$ im Alter von 75 bis 79 Jahren auf $50 \%$ ab einem Alter von 90 Jahren. $79 \%$ der Männer im Alter von 75 bis 79 Jahren nehmen zahnärztliche Leistungen in Anspruch, ab einem Alter von 90 Jahren nur noch $59 \%$ ([17]; - Abb. 1).

Mit zunehmender Gebrechlichkeit werden Zahnärztinnen oder Zahnärzte oftmals nur noch bei Beschwerden aufgesucht und der präventive Aspekt einer zahnmedizinischen Betreuung geht verloren $[18,19]$. Den ambulant
Pflegebedürftigen steht seltener als den allgemeinmedizinisch Gesunden ein chancengleicher Zugang zur zahnärztlichen Versorgung zur Verfügung [20]. Die Mundgesundheit tritt zunehmend in den Hintergrund [21-23], wodurch sich längerfristig der zahnärztliche Behandlungsbedarf erhöht. Zudem ist das Inanspruchnahmeverhalten zahnärztlicher Leistungen abhängig vom Vorhandensein einer Pflegestufe: Mehr als 2 Drittel $(68,2 \%)$ der äSoP gaben an, die Zahnärztin oder den Zahnarzt kontrollorientiert in Anspruch zu nehmen, wohingegen $61,2 \%$ der äSmP die Praxis nur beschwerdeorientiert aufsuchten ([5]; • Tab. 1).

Das Inanspruchnahmeverhalten von medizinischen Dienstleistungen wird durch verschiedene Faktoren geprägt. Der Zugang für Seniorinnen und Senioren zu ärztlichen und zahnärztlichen Leistungen stellt für die Sicherstellung von Chancengleichheit und Gerechtigkeit Ärztinnen und Ärzte sowie Zahnärztinnen und Zahnärzte gleichermaßen vor Herausforderungen und ethische Dilemmata [19, 24]. Im Bereich der
Seniorenzahnmedizin lassen sich Muster und Wirkungszusammenhänge von veränderbaren und unveränderbaren (Einfluss-)Faktoren, Ereignissen (z.B. Krankenhausaufnahme, Verlust naher Angehöriger, Übergang in eine Pflegesituation) und Lebensumständen (z.B. Abnahme der Eigenverantwortlichkeit, reduzierte Mobilität, steigende Morbidität, Armut), welche die Inanspruchnahme reduzieren oder sogar verhindern können, erkennen. Eine Herausforderung für die Akteure im Gesundheitswesen wird es zukünftig sein, sowohl in der Makro- als auch in der Mesoebene des Gesundheitswesens die Voraussetzungen dafür zu schaffen, dass auf der Mikroebene auch die heterogene Gruppe der älteren Menschen zahnärztlich einwandfrei und individuell versorgt werden kann. Beispielhaft seien ein vereinfachter Zugang zur Parodontaltherapie oder die Verordnung einer hochfluoridhaltigen Medikation für $\mathrm{SmP}$ zulasten der gesetzlichen Krankenkasse [25] genannt.

\section{Aufsuchende zahnmedizinische Betreuung}

In Deutschland sind unterschiedliche aufsuchende zahnmedizinische Versorgungskonzepte etabliert; diese reichen z. B. von einem einfachen Hausbesuch bei einem langjährigen Patienten bis hin zur vollstrukturierten Praxis mit mehreren Behandlerteams, die täglich SmP aufsuchen (•Tab. 5). Neben Eingangsuntersuchung und Therapieplanung besteht die Aufgabe der aufsuchenden Betreuung darin, die weiteren Behandlungsumstände (z.B. medizinische Diagnosen, Medikation, Vorhandensein von Angehörigen) und den Behandlungsort $\mathrm{zu}$ klären. Im Allgemeinen ist zu empfehlen, das Praxiskonzept strukturiert wachsen zu lassen. Auf diese Weise kann festgestellt werden, wie sich die Einflussfaktoren (z. B. Haltung der Leitungsebene zur Relevanz der Mundgesundheit von Bewohnerinnen und Bewohnern oder seniorenzugewandte Gesinnung der Mitarbeitenden in der Praxis) entwickeln und ob ein gemeinsames Wachsen an der Aufgabe möglich ist. Dabei sollte das gesamte Team Freude an der Betreuung der oft sehr dankbaren SmP empfinden. 


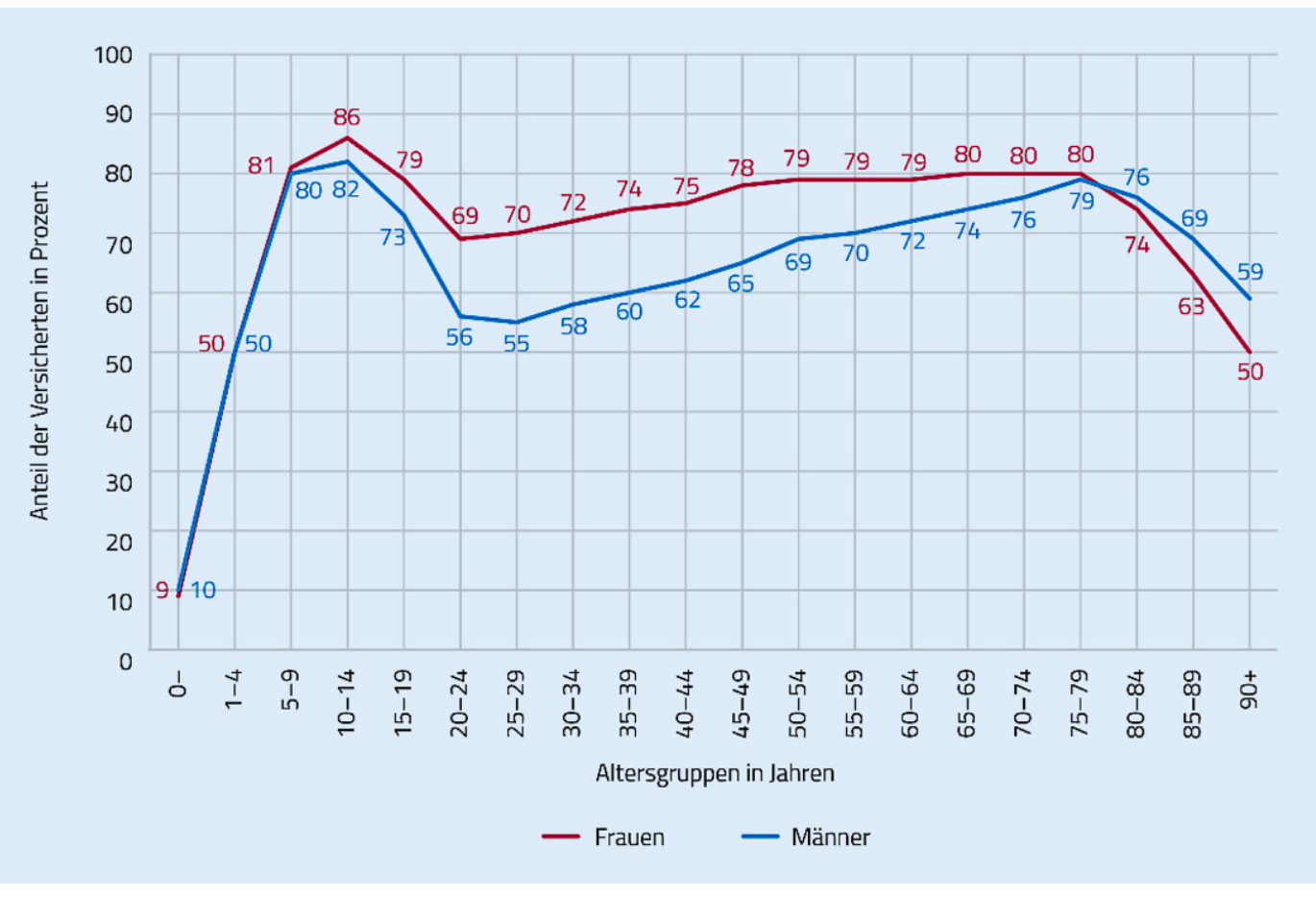

Abb. $1<$ Inanspruchnahme zahnärztlicher Leistungen nach Altersgruppen und Geschlecht im Jahr 2018. Anteil der Versicherten, die vertragszahnärztliche Leistungen der BEMA*Teile 1-5 in Anspruch nahmen [50]. (Mit freundlicher Genehmigung der (c) BARMER; *Einheitlicher Bewertungsmaßstab für zahnärztliche Leistungen gemäß $\S 87$ Abs. 2 und $2 \mathrm{~h}$ SGB V (BEMA))

Neue Gesetze (GKV-Versorgungsstrukturgesetz (GKV-VStG, in Kraft getreten 2012; [26]), Pflege-NeuausrichtungsGesetz (PNG, in Kraft getreten 2013; [27]) und GKV-Versorgungsstärkungsgesetz (GKV-VSG, in Kraft getreten 2015; [28])) bewirken eine bessere Honorierung des Aufwands bei der Versorgung von SmP und sind als Chance für die Betroffenen und die beteiligten Zahnmedizinerinnen und -mediziner zu verstehen. Weiterentwicklungen, z. B. auf Grundlage des A- und B-Konzeptes (Konzept zur vertragszahnärztlichen Versorgung von Pflegebedürftigen und Menschen mit Behinderungen) der wissenschaftlichen Fachverbände und der zahnmedizinischen Körperschaften [29], sind gemeinsam von der Gesetzgebung, Krankenversicherung, Patientenvertretung, Wissenschaft und Körperschaften $\mathrm{zu}$ veranlassen. Hier sollte zukünftig besonders die Versorgung in der Häuslichkeit im Vordergrund stehen, in der die meisten SmP leben. Sie entwickeln hier oft ein beschwerdeorientiertes Inanspruchnahmeverhalten, dessen negative Folgen oft erst in einer anschließenden stationären Pflegesituation auffallen.

\section{Vernetzung ambulanter und stationärer Krankenhaus- versorgung}

Eine weitere Herausforderung besteht in der Verbesserung der Vernetzung von ambulanten und stationären Versorgungsstrukturen.

\section{Kliniken für Geriatrie}

Während der Behandlung in den Kliniken für Geriatrie werden fast alle Körperteile und deren Funktionen untersucht, ggf. Therapiekonzepte erstellt und umgesetzt. Die Mundhöhle ist dabei jedoch fast immer ausgeschlossen, da Studierende der Humanmedizin während ihres Studiums nur wenig Berührungspunkte mit zahnmedizinischen Krankheitsbildern haben. Erkrankungen der Mundhöhle werden somit selten diagnostiziert. Zahnärztinnen und Zahnärzte sind in die stationäre geriatrische Versorgung nicht eingebunden [30-35]. Hauptgrund für diesen Sachverhalt ist, dass die erbrachten zahnärztlichen Leistungen bei einer Behandlung im stationären Setting nicht wie üblich über die kassenzahnärztliche Vereinigung mit der gesetzlichen Krankenkasse abgerechnet werden. Dies führt dazu, dass in aller Regel nur Notfallversorgungen stattfin- den. Da zahnärztliche Behandlungen nicht im stationären Abrechnungssystem abgedeckt sind und die abgebildeten kieferchirurgischen Leistungen nicht denjenigen entsprechen, die zur Versorgung von Patienten in den akutgeriatrischen Stationen benötigt werden, verstreicht häufig die Gelegenheit, geriatrische Patientinnen und Patienten auch über ihre Mundgesundheit aufzuklären, einen Weg zur wohnortnahen zahnärztlichen Behandlung anzubahnen und ggf. kleinere Maßnahmen mobil ausgerüstet sofort im Krankenhaus durchzuführen. Dies ist bedauerlich, bestünde doch beispielsweise die Chance, schwierige Extraktionen unter Antikoagulation mit ärztlicher Unterstützung während des Krankenhausaufenthaltes vorzunehmen und damit auch spätere kostenintensive Transporte zum Zahnarzt zu reduzieren. Es sollte demnach ein Weg gefunden werden, dass zahnärztliche Leistungen bei stationär aufgenommenen geriatrischen Patientinnen und Patienten mit den gesetzlichen Krankenversicherern abgerechnet werden können. 
Tab. 5 Merkmale aufsuchender Versorgungskonzepte der zahnärztlichen Praxis für Menschen mit Pflegebedarf. Einteilung nach dem Aufwand bei der Umsetzung (gering, mittel, hoch)

\begin{tabular}{|c|c|c|c|}
\hline $\begin{array}{l}\text { Merkmale der aufsu- } \\
\text { chenden zahnmedi- } \\
\text { zinischen Betreuung }\end{array}$ & $\begin{array}{l}\text { Umsetzung } \\
\text { mit geringem } \\
\text { Aufwand }\end{array}$ & $\begin{array}{l}\text { Umsetzung mit } \\
\text { mittlerem Aufwand }\end{array}$ & $\begin{array}{l}\text { Umsetzung mit } \\
\text { hohem Aufwand }\end{array}$ \\
\hline $\begin{array}{l}\text { Screening/ } \\
\text { Befundaufnahme }\end{array}$ & Durchführen & Durchführen & Durchführen \\
\hline Behandlungen & Keine & Einfache & (Fast) alle \\
\hline \multirow[t]{7}{*}{ Ausstattung } & Grundbesteck $^{\mathrm{a}}$ & Grundbesteck $^{\mathrm{a}}$ & Grundbesteck $^{\mathrm{a}}$ \\
\hline & \multirow[t]{6}{*}{ Fahrzeug } & Fahrzeug & Fahrzeug \\
\hline & & $\begin{array}{l}\text { Instrumente und Mate- } \\
\text { rialien entsprechend den } \\
\text { Behandlungen }\end{array}$ & $\begin{array}{l}\text { Instrumente und Mate- } \\
\text { rialien entsprechend den } \\
\text { Behandlungen }\end{array}$ \\
\hline & & $\begin{array}{l}\text { Kleine mobile Behand- } \\
\text { lungseinheit }\end{array}$ & $\begin{array}{l}\text { Kleine mobile Behand- } \\
\text { lungseinheit }\end{array}$ \\
\hline & & \multirow[t]{3}{*}{ Mobiles Ultraschallgerät } & $\begin{array}{l}\text { Oder praxisübliche zahn- } \\
\text { ärztliche Behandlungsein- } \\
\text { heit }\end{array}$ \\
\hline & & & $\begin{array}{l}\text { Evtl. zahnärztlicher mo- } \\
\text { biler Behandlungsstuhl }\end{array}$ \\
\hline & & & Mobiles Ultraschallgerät \\
\hline $\begin{array}{l}\text { Ort der Röntgendiagnos- } \\
\text { tik }\end{array}$ & Praxis & Praxis, selten mobil & Mobil \\
\hline \multirow{2}{*}{$\begin{array}{l}\text { Ort der Behandlung } \\
\text { bei einfachen Interven- } \\
\text { tionen, z. B. einfachen } \\
\text { Extraktionen }\end{array}$} & \multirow[t]{2}{*}{ Praxis } & Pflegeeinrichtung & Pflegeeinrichtung \\
\hline & & Häuslichkeit & Häuslichkeit \\
\hline \multirow{2}{*}{$\begin{array}{l}\text { Ort der Behandlung bei } \\
\text { schwierigen Interventio- } \\
\text { nen, z. B. bei mehreren } \\
\text { oder schwierigen Extrak- } \\
\text { tionen }\end{array}$} & \multirow[t]{2}{*}{ Praxis } & \multirow[t]{2}{*}{ Praxis } & Pflegeeinrichtung \\
\hline & & & $\begin{array}{l}\text { Häuslichkeit } \\
\text { (Selten Praxis) }\end{array}$ \\
\hline
\end{tabular}

\section{Zahnärztliche Behandlungen bei Abwehrverhalten von Patientinnen und Patienten}

Zahnärztinnen und Zahnärzte können der Herausforderung gegenüberstehen, dass eine Behandlung verbal oder schlussendlich auch durch Abwehrhandlungen verweigert wird. Besonders problematisch wird es z.B., wenn ein ehemals sanftmütiger Patient plötzlich ausgeprägt jähzornig wird, weil eine demenzielle Erkrankung Aggressionen mit sich bringt. Angst tritt dann als Grund für Wutanfälle in Erscheinung, wobei Menschen mit Demenz ein erhöhtes Risiko haben, sich selbst und andere zu gefährden [24, 36]. Zahnärztinnen und Zahnärzte, Angehörige und Pflegende sind hier in einer schwierigen Situation: Einerseits dürfen sie die Freiheitsrechte der betroffenen Person nicht unnötig einschränken, andererseits müssen sie zu deren Wohl handeln und dafür sorgen, dass die Mundpflege und zahnärztliche Behandlung möglichst aufrechterhalten bleibt. Freiheitseinschränkende Maßnahmen wie das Festhalten der betroffenen Person dürfen im Rahmen der Behandlung nur dann eingesetzt werden, nachdem alle anderen Möglichkeiten ausgeschöpft sind, um die Gefahr für die zu Behandelnden oder für Dritte $\mathrm{zu}$ verringern. Ferner ist $\mathrm{zu}$ berücksichtigen, dass eine zahnärztliche Behandlung einer festgehaltenen Person schon durch kleinste Bewegungen des Kopfes und die rotierenden Geräte im Mund ohne Verletzungen kaum möglich ist. Nur wenn das Risiko der Eigengefährdung oder der Gefährdung Dritter mit anderen Mitteln nicht in ausreichendem Maße vermeidbar erscheint, sollte eine zahnärztliche Behandlung in Intubationsnarkose (ITN) erfolgen [37, 38].
In der Regel bestehen Probleme bei der Umsetzung einer ambulanten Anästhesie für orale Sanierungen (z.B. Ort, Vergütung). Zunehmend sind Menschen mit Demenz und/oder mit zusätzlicher ausgeprägter Multimorbidität nicht mehr im Rahmen einer ambulanten Versorgung unter Sedierung oder ITN zahnärztlich $\mathrm{zu}$ versorgen. Vonseiten der Anästhesie kann oftmals eine etwaig auftretende Notfallsituation mit anschließender stationärer Behandlung und Beobachtung nicht ausgeschlossen werden. Dabei fällt eine stationäre Versorgungslücke für diese zahnärztlichen Behandlungen auf, wobei diese mit fachgerechter Nachsorge in den Kliniken für Geriatrie integriert werden könnten.

\section{Finanzierung des Mehraufwandes der zahnmedizinischen Betreuung}

Es ist eine Herausforderung, ältere Menschen mit Multimorbidität und Polymedikation zahnmedizinisch $\mathrm{zu}$ betreuen. Dafür sind zusätzliche Kenntnisse notwendig, die während des Studiums der Zahnmedizin gegenwärtig kaum adäquat gelehrt werden (können; [39]). Die wissenschaftliche Fachgesellschaft (Deutsche Gesellschaft für Alterszahnmedizin e.V.) bietet daher eine Spezialisierung in Seniorenzahnmedizin an [40], da die Etablierung und Koordination einer zahnmedizinischen Betreuung unter Einbindung des Praxisteams, der hausärztlichen Versorgung, des Pflegepersonals und der Angehörigen bei zunehmender Gebrechlichkeit komplex sind. Generell sind die zahnärztlichen Behandlungen zu den gleichen Bedingungen abrechenbar wie bei allgemeinmedizinisch gesunden Personen, obwohl der zeitliche Mehraufwand oftmals deutlich höher ist. Dies ist auch unter dem Umstand zu bewerten, dass SmP aufgrund ihrer Multimorbidität meist nicht mehr selbstständig regelmäßig eine zahnärztliche Praxis aufsuchen können. Zwischenzeitlich wurden bereits einige Verbesserungen hinsichtlich der Honorierung derartiger zahnärztlicher Leistungen geschaffen [26-28]. Diese reichen jedoch bis dato nicht aus, um eine genügende Anzahl an Zahnmedizinerinnen und -medizinern $\mathrm{zu}$ 
motivieren, auch für $\mathrm{SmP}$ auf fachlich hohem Niveau zur Verfügung zu stehen. Der Mehraufwand für die einzelnen zahnärztlichen Leistungen ist bisher nicht berücksichtigt. Dies unterstreicht, dass auf verschiedenen Ebenen die Voraussetzungen für eine verbesserte Inanspruchnahme zahnärztlicher Leistungen geschaffen werden sollten.

\section{Zahnmedizinisches Problem- bewusstsein in der Pflege}

\section{Allgemeine Situation}

Studien zeigen, dass das Pflegepersonal die Herausforderungen der Mundund Prothesenpflege aus Zeitgründen und aufgrund fehlenden theoretischen sowie praktischen Wissens nicht ausreichend erfüllen kann [21, 41-43]. In diesem Kontext sollten verschiedene Anknüpfungspunkte zwischen Pflege und Zahnmedizin in Aus-, Fort- und Weiterbildung weiterentwickelt werden [23, 44-46]. Die durch die gesetzliche Krankenversicherung finanzierten Kooperationsverträge zwischen zahnärztlichen Praxen und Pflegeeinrichtungen [47] ermöglichen den Zahnmedizinerinnen und -medizinern eine bessere Kommunikation und Darstellung der Mundsituation gegenüber der Pflege. In diesem Zusammenhang ist eine Vergütung für die Zahnmedizinerinnen und -mediziner, nicht aber für die Pflege vorhanden.

\section{Eine Chance: Expertenstandard Mundgesundheit}

Das Deutsche Netzwerk für Qualitätsentwicklung in der Pflege (DNQP) entwickelt seit 20 Jahren Expertenstandards (ES), die professionsübergreifend als ein anerkanntes Instrument zur Qualitätsentwicklung in der Pflege gelten [48]. Seit 2019 wird in Kooperation mit der Bundeszahnärztekammer, der Deutschen Gesellschaft für Alterszahnmedizin und der Arbeitsgemeinschaft Zahnmedizin für Menschen mit Behinderung oder besonderem medizinischen Unterstützungsbedarf ein neuer ES zur „Förderung der Mundgesundheit in der Pflege“ entwickelt. Im Gegensatz zu den anderen ES ist neben der Profession der Pflege erstmalig auch eine weitere Berufsgruppe, die Zahnmedizinerinnen und -mediziner, am Entwicklungsprozess beteiligt. Mit der Professionalisierung der Pflege steigt die Nachfrage nach ES kontinuierlich. Zusätzlich sind ES ein Bezugspunkt für die Qualitätsbeurteilung von Pflegeeinrichtungen durch den Medizinischen Dienst (Sozialgesetzbuch (SGB), elftes Buch (XI) - Soziale Pflegeversicherung; [49]). ES konkretisieren das aktuelle Wissen, wurden in der Vergangenheit von Gerichten herangezogen und haben auch für den Bereich des SGB, fünftes Buch (V) - Gesetzliche Krankenversicherung Relevanz. Es ist davon auszugehen, dass sich die Aufmerksamkeit der Pflege für Probleme in der Mundhöhle und der Mundpflege mit der Einführung des ES erhöhen wird. Klare Beschreibungen im Hinblick auf die Struktur-, Prozess- und Ergebnisqualität in der Pflege werden eine sehr gute Orientierung sowohl im Hinblick auf die interne Qualitätsentwicklung als auch auf die externe Qualitätssicherung bewirken. Der ES zur „Förderung der Mundgesundheit in der Pflege“ wird in die Ausbildung (auch Bachelor- und Masterstudiengänge) sowie Fort- und Weiterbildung im Pflegebereich einfließen. Die Zahnärztinnen und Zahnärzte sollten die Implementierung dieses ES als Chance wahrnehmen und seine Umsetzung unterstützen.

\section{Fazit}

Die zahnmedizinische Versorgung älterer Menschen steht vor der Herausforderung, dass sie zum Teil über Jahrzehnte sehr heterogene Lebensphasen mit unterschiedlichen zahnmedizinischen Ansprüchen begleiten muss. In der fitten Lebensphase gilt es, präventivzahnmedizinische Aufgaben und eine gesunde Mundhöhle sicherzustellen. In der gebrechlichen Lebensphase ist das Ziel, idealerweise zusammen mit der Hilfe von Angehörigen eine Mundsituation zu schaffen, die stabil und leicht zu pflegen ist. Diese Phase sollte engmaschig präventiv begleitet werden. Mit dem Auftreten von Pflegebedürftigkeit sind für die Zahnärztin oder den Zahnarzt
Ansprechpersonen zu etablieren, die mit Fachwissen die in den früheren Phasen hergestellte gute Mundsituation erhalten. Es ist wichtig, dass alle Akteure im Gesundheitswesen auch für die heterogene Gruppe der Seniorinnen und Senioren einen möglichst chancengleichen und barrierearmen Zugang zu einer zahnmedizinischen Versorgung schaffen. Das Gesundheitswesen mit seinen Akteuren in den Makro-, Meso- und Mikroebenen und seinen Strukturen sowie Schnittstellen ist gefordert, nicht nur eine zahnärztliche Behandlung, sondern eine zahnmedizinische Betreuung bereitzustellen, damit auch bei schwierigen Lebensumständen eine bestmögliche Mundgesundheit erreicht wird. Neben einer besseren Vernetzung ambulanter und stationärer Strukturen ist es eine Herausforderung, das Problembewusstsein innerhalb und außerhalb der zahnmedizinischen Berufsgruppen zu stärken und mundgesundheitsbezogene Aus-, Fort- und Weiterbildungsangebote zu etablieren.

\section{Korrespondenzadresse}

Prof. Dr. Ina Nitschke, MPH

Poliklinik für Zahnärztliche Prothetik und Werkstoffkunde, Universitätsklinikum Leipzig, Universität Leipzig

Liebigstraße 12, Haus 1, 04103 Leipzig,

Deutschland

ina.nitschke@medizin.uni-leipzig.de

Funding. Open Access funding enabled and organized by Projekt DEAL.

\section{Einhaltung ethischer Richtlinien}

Interessenkonflikt. I. Nitschke und S. Hahnel geben an, dass kein Interessenkonflikt besteht.

Alle beschriebenen Untersuchungen am Menschen wurden mit Zustimmung der zuständigen Ethikkommission, im Einklang mit nationalem Recht sowie gemäß der Deklaration von Helsinki von 1975 (in der aktuellen, überarbeiteten Fassung) durchgeführt. Von allen beteiligten Patientinnen und Patienten liegt eine Einverständniserklärung vor.

Open Access. Dieser Artikel wird unter der Creative Commons Namensnennung 4.0 International Lizenz veröffentlicht, welche die Nutzung, Vervielfältigung, Bearbeitung, Verbreitung und Wiedergabe in jeglichem Medium und Format erlaubt, sofern Sie den/die ursprünglichen Autor(en) und die Quelle ordnungsgemäß nennen, einen Link zur Creative Commons Lizenz 
beifügen und angeben, ob Änderungen vorgenommen wurden.

Die in diesem Artikel enthaltenen Bilder und sonstiges Drittmaterial unterliegen ebenfalls der genannten Creative Commons Lizenz, sofern sich aus der Abbildungslegende nichts anderes ergibt. Sofern das betreffende Material nicht unter der genannten Creative Commons Lizenz steht und die betreffende Handlung nicht nach gesetzlichen Vorschriften erlaubt ist, ist für die oben aufgeführten Weiterverwendungen des Materials die Einwilligung des jeweiligen Rechteinhabers einzuholen.

Weitere Details zur Lizenz entnehmen Sie bitte der Lizenzinformation auf http://creativecommons.org/ licenses/by/4.0/deed.de.

\section{Literatur}

1. Bundesinstitut für Bevölkerungsforschung Anteil der Altersgruppen unter 20 Jahren, ab 65 Jahre und ab 80 Jahre (1871-2060). https://www.bib.bund. de/Permalink.html?id=10203338. Zugegriffen: 8 . Jan. 2021

2. Die UN-Behindertenrechtskonvention Übereinkommen über die Rechtevon Menschen mitBehinderungen. http://www.behindertenbeauftragter. de//SharedDocs/Publikationen/UN_Konvention_ deutsch.pdf? _blob=publicationFile\&v=2. Zugegriffen: 8.Jan. 2021

3. Albrecht D, Mericske-Stern R, Nitschke I (2013) Besteht zahnärztlicher Behandlungsbedarf im akutgeriatrischen Krankenhaus? Z Senioren Zahnmed 1:151-158

4. Katsoulis J, Schimmel M, Avrampou M, Stuck AE, Mericske-Stern R (2012) Oral and general health status in patients treated in a dental consultation clinic of a geriatric ward in Bern, Switzerland. Gerodontology 29:602-610

5. Nitschke I, Micheelis W (2016) Krankheits- und Versorgungsprävalenzen bei Älteren Senioren mit Pflegebedarf. In: Jordan R, Micheelis W, Institut der Deutschen Zahnärzte (IDZ) (Hrsg) Fünfte Deutsche Mundgesundheitsstudie (DMS V). Deutscher Zahnärzte Verlag DÄV, Köln, S 557-578. ISBN 9783-7691-0020-4

6. Jordan RA, Bodechtel C, Hertrampf K, Hoffmann T, Kocher T, Nitschke I, Schiffner U, Stark H, Zimmer S, Micheelis W (2014) The Fifth German Oral Health Study (Fünfte Deutsche Mundgesundheitsstudie, DMS V) -rationale, design, and methods. BMC Oral Health 14:161. https://doi.org/10.1186/1472 6831-14-161

7. Lenz E (1999) Prävalenzen zu ausgewählten klinischen Variablen bei Senioren (65-74 Jahre): Zahnprothetischer Status bei den Senioren. In: Micheelis W, Reich E (Hrsg) Deutsche Mundgesundheitsstudie (DMS III). Ergebnisse, Trends und Problemanalysen auf der Grundlage bevölkerungsrepräsentativer Stichproben in Deutschland 1997. Deutscher Zahnärzteverlag DÄV, Cologne, S385-411

8. Kerschbaum T (2006) Zahnverlust und prothetische Versorgung. In: Micheelis W, Schiffner U, Institut der Deutschen Zahnärzte (Hrsg) Vierte Deutsche Mundgesundheitsstudie (DMS IV). Materialienreihe, Bd. 31. Deutscher Zahnärzte Verlag, Köln, S354-373

9. Nitschke I, Stark H (2016) Krankheits- und Versorgungsprävalenzen bei Jüngeren Senioren (65- bis 74-Jährige): Zahnverlust und prothetische Versorgung. In: Jordan R, Micheelis W, Institut der
Deutschen Zahnärzte (IDZ) (Hrsg) Fünfte Deutsche Mundgesundheitsstudie (DMS V). Deutscher Zahnärzte Verlag DÄV, Köln, S 416-451. ISBN 9783-7691-0020-4

10. Nitschke I, Stark H (2016) Krankheits- und Versorgungsprävalenzen bei Älteren Senioren (75bis 100-Jährige): Zahnverlust und prothetische Versorgung. In: Jordan R, Micheelis W, Institut der Deutschen Zahnärzte (IDZ) (Hrsg) Fünfte Deutsche Mundgesundheitsstudie (DMS V). Deutscher Zahnärzte Verlag DÄV, Köln, S 517-548. ISBN 9783-7691-0020-4

11. Bundesministerium für Gesundheit (2021) Geschäftsstatistik der Pflegekassen und der privaten Pflege-Pflichtversicherung zum 31.12.2019. https://www.bundesgesundheitsministerium.de/ fileadmin/Dateien/3_Downloads/Statistiken/ Pflegeversicherung/Zahlen_und_Fakten/ Zahlen_und_Fakten_der_SPV_Juli_2020_bf. pdf (Erstellt:01.2021).Zugegriffen: 8. Jan. 2021

12. Pflegestatistik 2017. https://www.destatis.de/ DE/Themen/Gesellschaft-Umwelt/Gesundheit/ Pflege/Publikationen/Downloads-Pflege/pflegedeutschlandergebnisse-5224001179005.xlsx? blob=publicationFile; Z Zugegriffen: 8 . Jan. 2021

13. Nitschke I, Hopfenmüller W (1996) Die Zahnmedizinische Versorgung älterer Menschen. [Dental care for older people].In:Mayer KU, Baltes PB (Hrsg) Die Berliner Altersstudie. [The Berlin Aging Study]. Akademie Verlag, Berlin, S429-448 (German)

14. Nitschkel, KunzeJ, HopfenmüllerW, ReiberT(2012) Die zahnmedizinische funktionelle Kapazität - ein Instrument in der Gerostomatologie. Quintessenz 63:207-210 (German)

15. Nitschke I, Wendland A, Weber $S$, Jockusch J, Lethaus B, Hahnel S (2021) Considerations for the prosthetic dental treatment of geriatric patients in Germany. J Clin Med 10:304. https://doi.org/10. 3390/jcm10020304

16. Nitschke I (2016) Krankheits- und Versorgungsprävalenzen bei Älteren Senioren (75- bis 100Jährige): Zahnmedizinische funktionelle Kapazität.In:Jordan R, Micheelis W, Institut derDeutschen Zahnärzte (IDZ) (Hrsg) Fünfte Deutsche Mundgesundheitsstudie (DMS V). Deutscher Zahnärzte Verlag DÄV, Köln, S 549-555. ISBN 978-3-76910020-4

17. Institut zur Gesundheitssystemforschung (2020) BARMER Zahnreport 2020. Schriften zur Gesundheitsanalyse Band 22. https://www.barmer.de/ blob/241616/3b39a6098e63921a427efe39809 32c16/data/dl-zahnreport2020-komplett.pdf.Zugegriffen: 8. Jan. 2021

18. Kiyak A, Reichmuth M (2005) Barriers to and enabler of older adults' use of dental services. JDentEduc 69:975-986

19. Nitschke I, Stillhart A, Kunze J (2015) Zur Inanspruchnahme zahnmedizinischer Dienstleistungen im Alter. Swiss Dent JSSO 125:475-490

20. Born G, Baumeister S, Sauer S et al (2006) Merkmale von Risikogruppen einer unzureichenden Inanspruchnahme zahnmedizinischer Leistungen - Ergebnisse der Study of Health in Pomerania (SHIP). Gesundheitswesen 68:257-264

21. Nitschke I, Hopfenmüller J, Hopfenmüller W (2012) Systematisches Review zur Frage der Mundgesundheit und des zahnmedizinischen Versorgungsgrades (stationäres Setting) bei pflegebedürftigen Menschen in Deutschland. In: Nitschke I, Schulte AG (Hrsg) Zur Mundgesundheit von Pflegebedürftigen und Menschen mit Behinderungen in Deutschland - eine systematische Übersicht (Review) auf der Grundlage aktueller Einzelstudien (2000-2012). IDZ-Information
3/2012. IDZ, Institut der Deutschen Zahnärzte, Köln

22. Shet R, Shetty S, Kalavathi M, Kumar MN, Yadav RD, Soumya S (2013) A study to evaluate the frequency and association of various mucosal conditions among geriatric patients. J Contemp Dent Pract 14:904-910

23. Zenthöfer A, Meyer-Kühling I, Hufeland AL, Schröder J, Cabrera T, BaumgartD, Rammelsberg P, Hassel AJ (2016) Carers' education improves oral health of older people suffering from dementia—Results of an intervention study. Clin Interv Aging 11:1755-1762

24. Nitschke I, Groß D, Kunze J (2017) Spezifische Bedarfe bei zahnärztlichen Patienten mit Demenz und ihre ethischen Implikationen. Ethik Med 29(1):71-86

25. Zimmer S, Bizhang M (2020) Fluoridprophylaxe in der Seniorenzahnmedizin. Z Senioren Zahnmed 3/2020:121-127

26. Bundesgesetzblatt Teil 1 Nr. 70 (2011): Gesetz zur Verbesserung der Versorgungsstruktur in der gesetzlichen Krankenversicherung (GKV-Versorgungsstrukturgesetz - GKV-VStG) https://www.bgbl.de/xaver/ $\mathrm{bgbl} /$ start.xav?startbk=Bundesanzeiger_ BGBI\&bk=Bundesanzeiger_BGBI\&start $=/ /^{*}$ \%5B@attr_id=\%27bgbl111s2983.pdf\%27 $\% 5 \mathrm{D} \#$ _bgbl_\%2F\%2F $\% 5 \mathrm{~B} \% 40$ attr id\%3D\%27bgbl111s2983.pdf\%27\%5D 1611037586574.Zugegriffen:8. Januar 2021

27. Bundesgesetzblatt Teil 1 Nr. 51 (2012) Gesetz zur Neuausrichtung der Pflegeversicherung (Pflege-NeuausrichtungsGesetz-PNG) https://www.bgbl.de/xaver/ $\mathrm{bgbl} /$ start.xav?startbk=Bundesanzeiger_ BGBI\&bk=Bundesanzeiger_BGBI\&start=//* \%5B@attr_id=\%27bgbl112s2246.pdf\%27 $\% 5 \mathrm{D} \#$ bgbl $\% 2 \mathrm{~F} \% 2 \mathrm{~F} * 5 \mathrm{~B} \% 40$ attr id\%3D\%27bgbl112s2246.pdf\%27\%5D_ 1611038879151.Zugegriffen: 8. Januar 2021

28. Bundesgesetzblatt Teil 1 Nr. 30 (2015) Gesetz zur Stärkung der Versorgung in der gesetzlichen Krankenversicherung (GKV-Versorgungsstärkungsgesetz - GKV-VSG) https://www.bgbl.de/xaver/ $\mathrm{bgbl} /$ start.xav?startbk=Bundesanzeiger_BGBI\& jumpTo=bgbl115s1211.pdf\#_bgbl_\%2F\%2F* \%5B\%40attr_id\%3D\%27bgbl115s1211.pdf\%27 \%5D_1611039203384 Zugegriffen: 8. Januar 2021

29. Bundeszahnärztekammer und Kassenzahnärztliche Bundesvereinigung (2010) Mundgesund trotz Handicap und hohem Alter. Konzept zur vertragszahnärztlichen Versorgung von Pflegebedürftigen und Menschen mit Behinderungen ( $\mathrm{A}$ - und B-Konzept). https://www.bzaek.de/fileadmin/PDFs/ presse/AuB_Konzept.pdf. Zugegriffen: 10. Jan. 2021

30. Asher R, PolakD, Assad R, Goldstein M (2019) Knowledge and opinion of internists and gynecologists physicians on periodontal medicine. Oral Health Prev Dent 17:251-256

31. Barbe G, Kunter H, Noack MJ, Barbe AG (2020) Do subjectively and objectively impaired oral health parameters influence geriatric assessment results in hospitalized geriatric patients? Eur Geriatr Med 11:465-474

32. Baudermann C (2015) Untersuchung zur Zahnund Mundgesundheit stationär betreuter älterer Patienten in der Akutgeriatrie und Analyse des sich daraus ergebenden Beratungs- und Behandlungsbedarfs. Dissertation an der RuprechtKarls-Universität Heidelberg. http://www.ub.uni- 
heidelberg.de/archiv/22787. Zugegriffen: 8. Jan. 2021

33. Maeda K, Mori N (2020) Poor oral health and mortality in geriatric patients admitted to an acute hospital:an observational study. BMC Geriatr 20:26

34. Sjögren $\mathrm{P}$, Wårdh I, Zimmerman $\mathrm{M}$, Almståhl $\mathrm{A}$, Wikström M (2016) Oral care and mortality in older adults with pneumonia in hospitals or nursing homes: systematic review and meta-analysis. J Am Geriatr Soc 64(10):2109-2115

35. Taguchi A, Shiraki M, Toshitsugu S, Ohta H, Soen S, Japan Osteoporosis Society (2016) Lack of cooperation between physicians and dentists during osteoporosis treatment may increase fractures and osteonecrosis of the jaw. Curr Med Res Opin 32:1261-1268

36. Handley M, Bunn F, Goodman C (2017) Dementiafriendly interventions to improve the care of people living with dementia admitted to hospitals: a realist review. BMJOpen 7(7):e15257. https://doi. org/10.1136/bmjopen-2016-015257

37. Jockusch J, Sobotta BAJ, Nitschke I (2020) Outpatient dental care for people with disabilities under general anaesthesia in Switzerland. BMC Oral Health 20(1):225. https://doi.org/10.1186/ s12903-020-01203-6

38. Jockusch J, Hopfenmüller W, Ettinger R, Nitschke I (2021) Outpatient, dental care of adult vulnerable patients under general anaesthesia - a retrospective evaluation of need for treatment and dental follow-up care. Clin Oral Investig 25(4):2407-2417. https://doi.org/10.1007/s00784-020-03564-2

39. Nitschke I, Neitzel M, Sobotta BAJ (2018) Development of undergraduate gerodontology courses in Austria, Germany and Switzerland 2004-2014. Eur J Dent Educ 22(3):e303-e311. https://doi.org/10. 1111/eje.12294

40. Deutsche Gesellschaft für Alterszahnmedizin (2017) Spezialisierungsordnung. https:// dgaz.org/images/downloads/oeffentliche_ Downloads/Zertifizierung/2017_August_ Zertifizierungsordnung.pdf. Zugegriffen: 8 . Jan. 2921

41. Nitschkel, Majdani M, Sobotta B et al (2010) Dental care of frail older people and those caring for them JClin Nurs 19(13-14):1882-1890

42. Wong FMF, Ng YTY, Leung WK (2019) Oral health and its associated factors among older institutionalized residents-a systematic review. Int J Environ Res Public Health 16(21):4132-4126

43. Anderson RA, Wang J, Plassman BL, Nye K, Bunn M, Poole PA, Drake C, Xu H, Ni Z, Wu B (2019) Working together to learn new oral hygiene techniques: Pilot of a carepartner-assisted intervention for persons with cognitive impairment. Geriatr Nurs 40(3):269-276. https://doi.org/10.1016/j. gerinurse.2018.11.002

44. Hoben M, Clarke A, Huynh KT, Kobagi N, Kent A, $\mathrm{Hu} \mathrm{H}$, Pereira RAC, Xiong T, Yu K, Xiang H, Yoon MN (2017) Barriers and facilitators in providing oral care to nursing home residents, from the perspective of care aides: a systematic review and meta-analysis. Int J Nurs Stud 73:34-51. https://doi.org/10.1016/ j.ijnurstu.2017.05.003

45. Estes KR, CallananD, Rai N,PlunkettK, Brunson D, TiwariT(2018) Evaluation of an Interprofessional Oral Health Assessment Activity in Advanced Practice Nursing Education. JDent Educ 82(10):1084-1090. https://doi.org/10.21815/JDE.018.103

46. Barbe AG, Kottmann HE, Müller D, Simic D, Derman SHM, Wicht MJ, Noack MJ (2019) Evaluation of time and resources required for professional dental cleaning in nursing home residents. Spec Care Dentist 39(2):89-96. https:// doi.org/10.1111/scd.12352

47. Kassenzahnärztlichen Bundesvereinigung, GKVSpitzenverband (2019) Bericht der Kassenzahnärztlichen Bundesvereinigung und des GKV-Spitzenverbands an die Bundesregierung zur Entwicklung der kooperativen und koordinierten zahnärztlichen und pflegerischen Versorgung von pflegebedürftigen Versicherten in stationären Pflegeeinrichtungen gem. § 119b Abs. 3 Satz 3 SGB V; https://www. gkv-spitzenverband.de/media/dokumente/ krankenversicherung_1/zahnaerztliche versorgung/zae_sonstige_vereinbarungen 1/ZA_Pflege_Bericht-bundesreg-119b-SGBV2019-06-30-final.pdf Zugegriffen am 8. Januar 2021

48. de Pflege-online, Fachmedien S (2021) Diese zehn Expertenstandards sollten Sie kennen. https://www.pflegen-online.de/diese-zehnexpertenstandards-sollten-sie-kennen. Zugegriffen: 8. Jan. 2021

49. Medizinischer Dienst des Spitzenverbandes Bund der Krankenkassen e.V. (MDS), Medizinischer Dienst des GKV-Spitzenverbande (2020im) Expertenstandard nach § 113a SGB XI „Erhaltung und Förderung der Mobilität in der Pflege" Aktualisierung $2020 \mathrm{im}$ Auftrag der Vertragsparteien nach $\S 113$ Abs. 1 SGB XI vertreten durch den Verein Geschäftsstelle Qualitätsausschuss Pflege e.V. https://www.mds-ev.de/fileadmin/dokumente/ Publikationen/SPV/Expertenstandards 113/ Expertenstandard_Erhaltung_Foerderung_ Mobilitaet_Pflege_201119.pdf. Zugegriffen: 8 . Jan. 2021

50. Institut zur Gesundheitssystemforschung (2020) Infografik zum BARMER Zahnreport 2020 Schriften zur Gesundheitsanalyse, Band 22, Institut zur Gesundheitssystemforschung. https:// www.barmer.de/presse/infothek/studien-undreports/zahnreporte/infografiken-zahnreport2020-241608. Zugegriffen: 11. Febr. 2021 